\title{
Chemically distinct nuclei and circumnuclear rings in lenticular galaxies NGC 4429 and NGC 7013*
}

\author{
O. K. Sil'chenko ${ }^{1,2,3}$ and V. L. Afanasiev ${ }^{4}$ \\ 1 Sternberg Astronomical Institute, University Av. 13, Moscow 119899, Russia \\ ${ }^{2}$ Isaac Newton Institute of Chile, Moscow Branch \\ 3 UK Astronomy Data Centre, Guest Investigator \\ 4 Special Astrophysical Observatory, Nizhnij Arkhyz 369167, Russia
}

Received 12 September 2001/ Accepted 13 December 2001

\begin{abstract}
The central regions of the regular lenticular galaxies NGC 4429 and NGC 7013 have been studied with the Multi-Pupil Field (Fiber) Spectrograph (MPFS) of the $6 \mathrm{~m}$ telescope of the Special Astrophysical Observatory RAS (Nizhnij Arkhyz, Russia) in two spectral ranges, the blue one including the strong absorption lines Mg I and $\mathrm{Fe} \mathrm{I}$ and the red one including the emission lines $\mathrm{H} \alpha$ and $[\mathrm{N} \mathrm{II}] \lambda 6583$. We confirm the presence of the chemically distinct nucleus in NGC 7013 reported earlier. Besides this unresolved (in the magnesium index) structure, we have found a ring of younger stellar populations than those in the nucleus and in the bulge; the radius of this ring is about $6^{\prime \prime}(400 \mathrm{pc})$. A similar ring, distinguished by high magnesium- and iron-index values and bordered by $\mathrm{H} \alpha$ emission at its inner edge, with a radius of $6^{\prime \prime}(500 \mathrm{pc})$, is found in NGC 4429 . We try to relate the ringed structure of the chemically decoupled cores in these galaxies with past, now dissolved, large-scale bars whose remnants are now seen in NGC 4429 and NGC 7013 as lenses between the bulges and global disks. An analysis of the gas and star kinematics in the centers of the galaxies has revealed the presence of an inclined circumnuclear disk in NGC 7013 and the existence of minibar in NGC 4429.
\end{abstract}

Key words. galaxies: individual: NGC 4429 - galaxies: individual: NGC 7013 - galaxies: nuclei galaxies: stellar content - galaxies: kinematics and dynamics - galaxies: evolution

\section{Introduction}

Since 1992 (Sil'chenko et al. 1992) we have studied chemically distinct stellar nuclei in normal early-type disk galaxies and have tried to guess their origin. As these nuclei often harbour stellar populations that are much younger on average than those of their surrounding bulges and as they are chemically more evolved, being more metal-rich, we conclude that they were formed in some secondary starformation bursts which took place in the galactic centers well after the main galactic components, the bulges first of all, finished their formation. However we do not know exactly what sudden event provoked the secondary starformation bursts. It is clear that an abrupt intense gas inflow into the center of a galaxy is able to stimulate such

Send offprint requests to: O. K. Sil'chenko,

e-mail: olga@sai.msu.su

* Based on observations collected with the $6 \mathrm{~m}$ telescope of the Special Astrophysical Observatory (SAO) of the Russian Academy of Sciences (RAS) which is operated under the financial support of Science Department of Russia (registration number 01-43) and on data from the HST and ING Archives. a burst; but it is also clear that radial gas re-distribution may result from various causes. It may be external action, such as tidal interaction with a by-passing galaxy (e.g. Noguchi 1988), or a minor merger (e.g. Thakar \& Ryden 1998), or it may be an internal instability of a global gaseous disk caused by a transient bar (e.g. Heller \& Shlosman 1994). In any case, other imprints on the global galactic structure and kinematics must remain from such a catastrophic event, and our task is to find them and to relate them to the presence of chemically distinct nuclei.

Up to now we have had several successes in this way. We have found that kinematically detached subsystems in galaxies, including global ones, almost always signify the presence of chemically distinct nuclei: the nuclei have appeared to be distinct in the galaxies with polar (NGC 2841, NGC 6340, NGC 7217, NGC 7280) or counterrotating (NGC 4826, NGC 4138, NGC 7332) circumnuclear ionized gas, as well as in 4 of 5 known disk galaxies with a global stellar counterrotating component (NGC 2841, NGC 4138, NGC 7217, and NGC 7331). Besides this unambiguous finding, we believe there is a relation between the chemically distinct nuclei and the 
multi-tier structure of global stellar disks in NGC 615, NGC 5533, NGC 7217, and NGC 7331; perhaps, the rare phenomenon of exponential-profile bulges in early-type disk galaxies also has a common origin with the chemically distinct central populations (NGC 4138, NGC 7217, ...). Now we suggest to investigate nuclear and circumnuclear stellar populations in galaxies with another peculiar feature in the global structure - exactly, in the galaxies with stellar rings. The inner stellar rings are usually thought to be consequences of gas accumulation in loci of Inner Lindblad resonances of a global bar (e.g. Buta 1993; Buta \& Crocker 1993), with subsequent intense star formation. However, many cases are known where there are rings but there is no bar in a galaxy. Particularly, the case of NGC 7217 where there are three different-scale rings but no bar has been analysed in detail by Buta et al. (1995). They have concluded that an extended stellar halo of the galaxy is slightly triaxial, thus being capable of producing resonances. It seems probable that NGC 7217 has had a global bar in the past, but now the bar has dissolved. Transient bars are very promising for a secular evolution of galaxies because they can leave a central gas concentration behind them, including inclined circumnuclear disks (Friedli \& Benz 1993), counterrotating stellar components (Athanassoula 1996; Tremaine \& Yu 2000), and, of course, rings. Athanassoula (1996) noticed that rings were much more long-lived than the bars that produced them, so they may remain detectable even after bar dissolution, becoming signatures of the past bar existence. In this work we consider NGC 4429 and NGC 7013, both of the very early type, with rings but without bars. The global characteristics of the galaxies are given in Table 1 . Previously we claimed the presence of a chemically distinct nucleus in NGC 7013 from our early MPFS observations (Sil'chenko et al. 1992). NGC 4429 has been put into our list of candidates for possessing such nuclei (Sil'chenko 1994) due to its prominently red center. We present the results on the stellar population properties and gas and stellar kinematics within the radius of $\sim 8^{\prime \prime}$.

\section{Observations, data reduction, and archive data}

The spectral data that we analyse in this work were obtained with two different integral-field spectrographs. Integral-field spectroscopy is a rather new approach that was first proposed by Courtes some 15 years ago - for a description of the instrumental idea see e.g. Bacon et al. (1995). It allows one to simultaneously obtain a set of spectra in a broad spectral range from an extended area on the sky, for example, from a central part of a galaxy. A 2D array of microlenses provides a set of micropupils which are put into the entry of a spectrograph, and after having reduced the full set of spectra corresponding to the individual spatial elements, we obtain a list of fluxes in continuum and in emission lines, of line-of-sight velocities, both for stars and ionized gas, and of absorption-line equivalent widths which are usually expressed as indices in the well-formulated Lick system (Worthey et al. 1994).
This list can be transformed into two-dimensional maps of the abovementioned characteristics for the central part of a galaxy that is studied. Besides the panoramic view benefits, such an approach gives a unique opportunity to overlay various $2 \mathrm{D}$ distributions without any difficulties with positioning. In our work we usually observe two spectral ranges - the green one, 4300-5600 $\AA$, and the red one, 6200-6900 $\AA$. The green spectra are used to calculate the Lick indices $\mathrm{H} \beta, \mathrm{Mgb}$, Fe5270, and Fe 5335 which are suitable to determine metallicity, age, and $\mathrm{Mg} / \mathrm{Fe}$ ratio of old stellar populations (Worthey 1994), and also are used for cross-correlation with the spectrum of a template star, usually of K0III-K3III spectral type, to obtain in such a way a line-of-sight velocity field for the stellar component and a map of stellar velocity dispersion. The red spectral range contains emission lines $\mathrm{H}_{\alpha}$ and [N II] $\lambda 6583$, the strongest ones in the optical, which are used to derive a line-of-sight velocity field for the ionized gas. The list of the exposures made for NGC 4429 and NGC 7013 with two different $2 \mathrm{D}$ spectrographs is given in Table 2 . The examples of spectra are shown in Figs. 1 and 2.

The observations earlier than 1998 were made with the old variant of the Multi-Pupil Field Spectrograph (MPFS) of the $6 \mathrm{~m}$ telescope (Afanasiev et al. 1996). It included two modes, with $8 \times 12$ or $8 \times 16$ micropupil arrays, supported respectively by CCD detectors of $520 \times 580$ and $1040 \times 1160$ pixel formats. The spatial scale was $1^{\prime \prime} 3$ per microlens. The reciprocal dispersion was $1.6 \AA$ per pixel with the spectral resolution of 4-6 $\AA$ slightly varying over the frame. To calibrate the wavelength scale, we exposed separately a spectrum of the hollow cathode lamp filled with helium, neon, and argon; an internal accuracy of linearization was typically $0.25 \AA$ in the green and $0.1 \AA$ in the red, but we checked the accuracy and an absence of systematic velocity shift by measuring strong emission lines of the night sky $[\mathrm{O} \mathrm{I}] \lambda 5577$ and $[\mathrm{O} \mathrm{I}] \lambda 6300$. In this variant of $2 \mathrm{D}$ spectroscopy, we also exposed separately a blank area of the sky some arcminutes from the galaxy, because we needed sky subtraction to properly calculate the equivalent widths of the absorption lines. The system of Lick indices was checked by observing 9 standard stars from the list of Worthey et al. (1994); we found that our index system coincided with the Lick one within a mean deviation of $0.05 \AA$.

Later in 1998 a new variant of the MPFS became operational in the prime focus of the $6 \mathrm{~m}$ telescope (http://www.sao.ru/ gafan/ devices/ mpfs/).

With respect to the old one, in the new variant of the MPFS the field of view is increased and the common spectral range is larger due to using fibers: they transmit light from $16 \times(15 \div 16)$ square elements of the galaxy image to the slit of the spectrograph together with additional 16 fibers that transmit the sky background light taken apart from the galaxy, so separate sky exposures are not necessary now. A flat-field light is passed through all the fibers so the transmittence variations of the fibers are also corrected by flat-fielding. The size of one spatial element is approximately $1^{\prime \prime} \times 1^{\prime \prime}$; a CCD TK $1024 \times 1024$ detector 
Table 1. Global parameters of the galaxies.

\begin{tabular}{lll}
\hline NGC & 4429 & 7013 \\
\hline Type (NED) & $\mathrm{SA}(\mathrm{r}) 0+$ & $\mathrm{SA}(\mathrm{r}) 0 / \mathrm{a}$ \\
$R_{25}, \mathrm{kpc}(\mathrm{LEDA})$ & 12.7 & 8.1 \\
$B_{\mathrm{T}}^{0}$ (LEDA) & 10.71 & 11.36 \\
$M_{B}$ (LEDA) & -20.23 & -19.23 \\
$B-V(\mathrm{RC} 3)$ & 0.96 & 0.71 \\
$U-B(\mathrm{RC} 3)$ & 0.54 & 0.19 \\
$V_{\mathrm{r}}(\mathrm{NED}), \mathrm{km} \mathrm{s}{ }^{-1}$ & 1106 & 779 \\
Distance, Mpc & $17(\mathrm{Virgo})$ & $13\left(\mathrm{LEDA}, H_{0}=75 \mathrm{~km} \mathrm{~s}^{-1} \mathrm{Mpc}^{-1}\right)$ \\
Inclination $(\mathrm{LEDA})$ & $75^{\circ}$ & $90^{\circ}$ \\
PA & $99^{\circ}$ & $157^{\circ}$ \\
$v_{\mathrm{m}}, \mathrm{km} \mathrm{s}^{-1}$ (LEDA) & - & 150 \\
$\sigma_{*}, \mathrm{~km} \mathrm{~s}^{-1}$ (LEDA) & 221 & 90 \\
\hline
\end{tabular}

Table 2. 2D spectroscopy of NGC 4429 and NGC 7013.

\begin{tabular}{llllllcc}
\hline Date & Galaxy & Exposure & Configuration & Field & Scale, ${ }^{\prime \prime} /$ lense & Seeing & Sp. range \\
\hline 20.08 .96 & NGC 7013 & $90 \mathrm{~min}$ & MPFS+CCD $520 \times 580$ & $13^{\prime \prime} \times 19^{\prime \prime}$ & 1.6 & 2.6 & $6200-6800 \AA$ \\
10.10 .96 & NGC 7013 & $60 \mathrm{~min}$ & MPFS+CCD $1040 \times 1160$ & $11^{\prime \prime} \times 21^{\prime \prime}$ & 1.3 & $1^{\prime \prime} .8$ & $4400-5400 \AA$ \\
09.05 .97 & NGC 4429 & $50 \mathrm{~min}$ & MPFS+CCD $1040 \times 1160$ & $11^{\prime \prime} \times 21^{\prime \prime}$ & 1.3 & $1^{\prime \prime} 5$ & $6100-7100 \AA$ \\
17.08 .98 & NGC 7013 & $60 \mathrm{~min}$ & MPFS+CCD $1024 \times 1024$ & $16^{\prime \prime} \times 16^{\prime \prime}$ & 1.0 & $2^{\prime \prime} 0$ & $4250-5600 \AA$ \\
14.06 .99 & NGC 4429 & $60 \mathrm{~min}$ & MPFS+CCD $1024 \times 1024$ & $16^{\prime \prime} \times 16^{\prime \prime}$ & 1.0 & $1^{\prime \prime} 5$ & $4270-5600 \AA$ \\
\hline
\end{tabular}

is used. The reciprocal dispersion is $1.35 \AA$ per pixel, with a rather stable spectral resolution of $5 \AA$. The wavelength calibration is made in a way similar to the previous variants of the MPFS observations. To calibrate the new MPFS index system onto the standard Lick one, we observed 15 stars from the list of Worthey et al. (1994) during four observational runs; the comparison of the indices calculated directly from the new MPFS spectra with those tabulated in Worthey et al. (1994) has allowed us to derive linear relations that are now used to reduce the indices into the standard Lick system. To correct the index measurements for the stellar velocity dispersion, that is usually substantially non-zero in the centers of early-type galaxies, we have smoothed the spectrum of the standard star HD 97907 by a set of Gaussians of various widths; the derived dependencies of index corrections on $\sigma$ were approximated by polynomials of 4th order and applied to the measured index values before their calibrations into the Lick system. The typical index correction for the stellar velocity dispersion is about $0.4 \AA$ at $\sigma=200 \mathrm{~km} \mathrm{~s}^{-1}$.

The exposure times for the galaxies observed with both variants of the MPFS were chosen to be long enough to provide a signal-to-noise ratios of more than 60 (per Angström) in the nucleus and $\leq 20$ near the edges of the frames; the corresponding random error estimations made in the manner of Cardiel et al. (1998) range from $0.15 \AA$ in the center to $1 \AA$ for the individual spatial elements in the outer part. To keep a constant level of accuracy along the radius, besides constructing $2 \mathrm{D}$ maps of indices, we co-added also the elementary spectra obtained with every 2D spectrograph in concentric rings centered on the nuclei and studied the radial dependencies of the stellar population properties by comparing the absorption-line indices observed by us to evolutionary synthesis models of old stellar populations of Worthey (1994) and Tantalo et al. (1998). We estimate the typical accuracy of our azimuthally averaged indices as $0.1 \AA$.

Besides the 2D spectral data, we use also some archive photometric data. Both galaxies were observed by HST. NGC 4429 has been exposed with WFPC2 on July 24, 1994, through the F606W filter for $80+80 \mathrm{~s}$, in the program "The nuclei of nearby S0 and spiral galaxies" (ID 5446, PI G. Illingworth). NGC 7013 was exposed with WFPC2 on August 17, 1996, also through the F606W filter, but for $400+200 \mathrm{~s}$, and with NICMOS, 2nd camera, on August 14, 1997, through the F160W filter for $128 \mathrm{sec}$; both observations were made in the programs of M. Stiavelli on the bulges of spiral galaxies. To expand our photometric analysis to outer parts of the global galactic disks, we use also ground-based photometry. NGC 7013 was viewed at La Palma Jacobus Kapteyn Telescope, on May 18, 1998, for $20 \mathrm{~min}$, through the $R$-filter, under the seeing of $1^{\prime \prime} 2$; we have retrieved these data from the ING Archive. For NGC 4429 we use the public data of Frei et al. (1996), obtained at the Lowell $1.1 \mathrm{~m}$ telescope through the $B$ - and $R$-filters: though of low resolution (seeing of $3^{\prime \prime}-4^{\prime \prime}$ ), these data are deep and well-calibrated. 

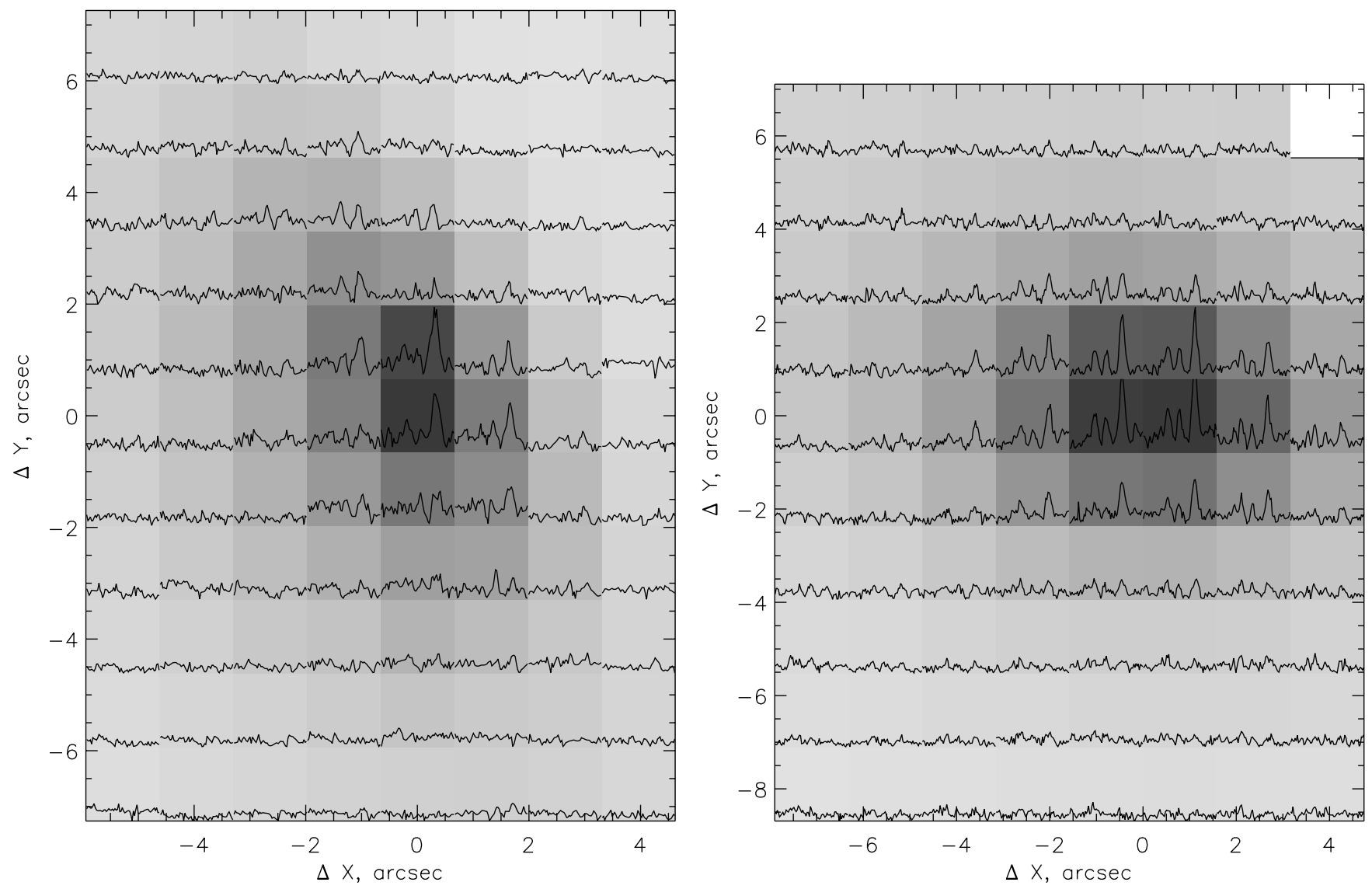

Fig. 1. Examples of the spectral data cubes in the red spectral range 6530-6630 $\AA$ are displayed against the gray-scaled background of the red continuum intensity; the most prominent emission line is $[\mathrm{NII}] \lambda 6583$. The left plot represents NGC 4429 , the right one NGC 7013.

All the data, spectral and photometric, except the data obtained with the new MPFS, were reduced with the software produced by Dr. V. V. Vlasyuk at the Special Astrophysical Observatory (Vlasyuk 1993). Primary reduction of the data obtained with the new MPFS was done in IDL with software created by one of us (V.L.A.). The Lick indices were calculated with our own FORTRAN program as well as by using the FORTRAN program of Dr. A. Vazdekis.

\section{Nuclear and circumnuclear stellar populations}

\subsection{NGC 7013}

In our earlier work made with the first fibre variant of the MPFS equipped with a photon-counting system we claimed the presence of a chemically distinct nucleus in NGC 7013 (Sil'chenko et al. 1992). Now, when the accuracy of absorption-line index measurements has been greatly improved due to CCDs, we confirm this finding; moreover, now we can add new details concerning the configuration of the chemically (and evolutionary) decoupled central region. Figure 3 presents two-dimensional maps of index distributions according to the best data of August 1998. The magnesium index is peaked in the very center, and its distribution looks quasi-axisymmetric. The iron index also has its maximum in the center, with a gradual decrease of $\langle\mathrm{Fe}\rangle \equiv(\mathrm{Fe} 5270+\mathrm{Fe} 5335) / 2$ along the radius; but this image is not so symmetric, and the whole iron-enhanced region seems to be more extended than the magnesium-enhanced one. Such differences between the magnesium and the iron index distributions are typical for chemically distinct cores in disk galaxies according to our modest statistics. But what is quite unusual is the map of the $\mathrm{H} \beta$-index: it demonstrates a ring-shaped enhancement at a radius of about $5^{\prime \prime}$. This is for the first time that we find a minimum of the absorption-line index in the nucleus.

Figure 4 presents azimuthally averaged profiles of the absorption-line indices that aim to help us quantify the picture implied by Fig. 3. First of all, the comparison of the independent observations of October 1996 and of August 1998, which were made with different variants of the MPFS, proves the accuracy of the azimuthallyaveraged indices of $0.1 \AA$ : though the profiles from the old MPFS are less prolonged because of its smaller field of view, within $R=5^{\prime \prime}$ the agreement of both sets is excellent. Both metal-line indices show a drop by $0.5 \div 0.6 \AA$ between the nucleus and the circumnuclear region (bulge?); but the magnesium-enhanced nucleus is unresolved under 

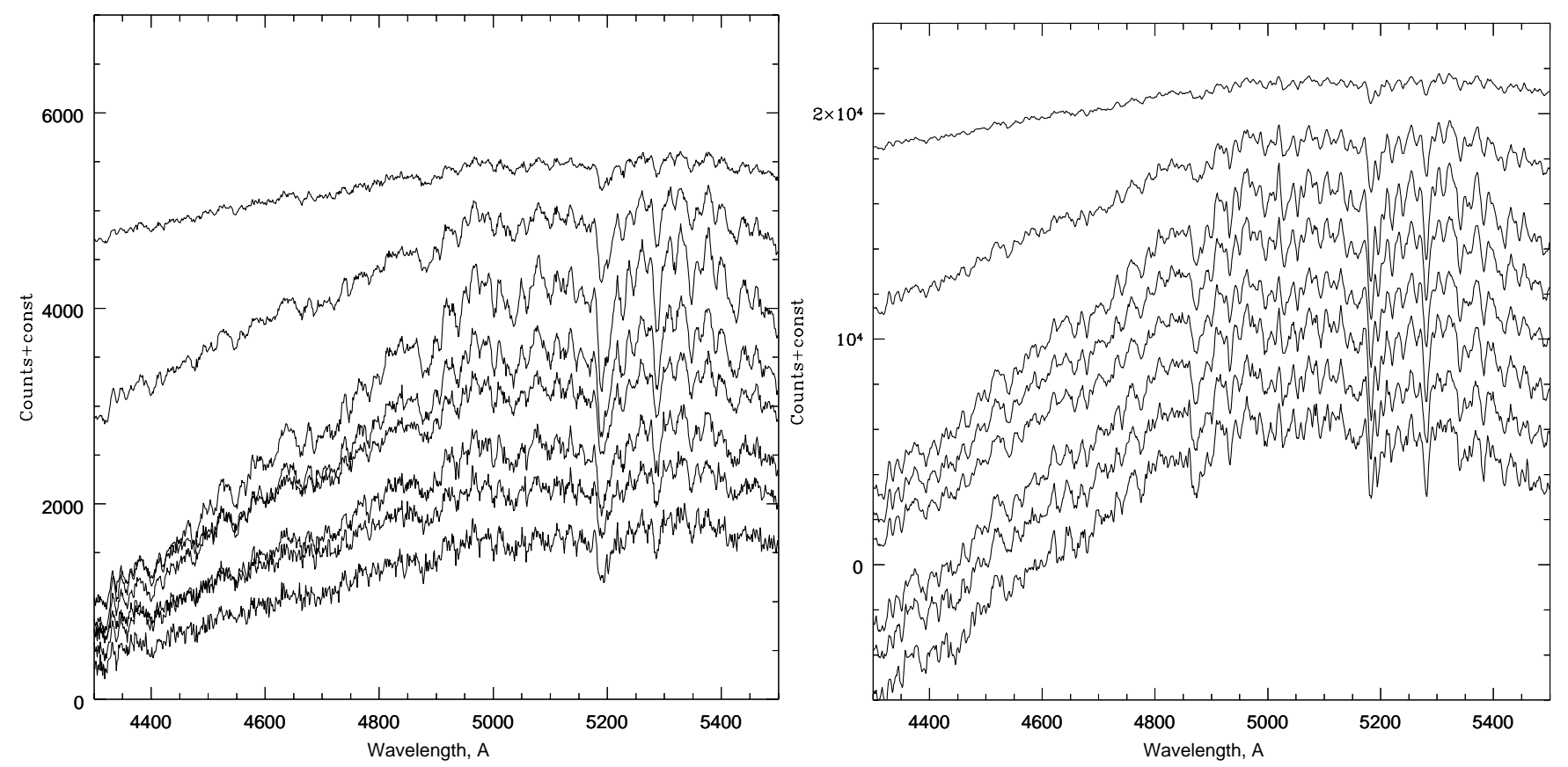

Fig. 2. Examples of the azimuthally averaged green spectra: the left plot is the observations of NGC 4429 in June 1999 , the right plot is the observations of NGC 7013 in August 1998. The spectra are shifted along the $y$-axis by an arbitrary constant for illustrative purposes; the uppermost spectra belong to the nuclei, the next spectra from top to bottom are taken with a step of $1^{\prime \prime}$ along the radius.
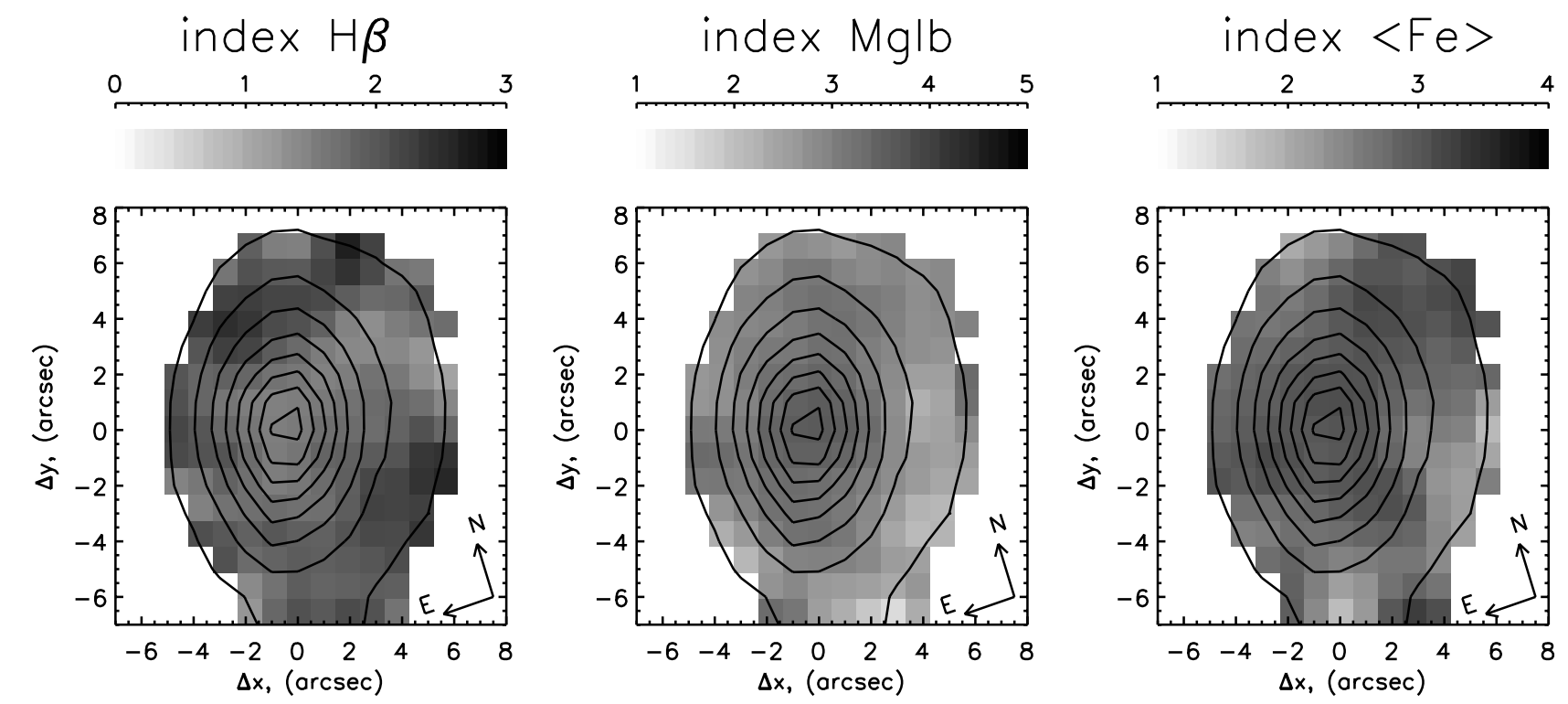

Fig. 3. Lick index maps for the central part of NGC $7013 ;<\mathrm{Fe}>\equiv(\mathrm{Fe} 5270+\mathrm{Fe} 5335) / 2$.

our seeing conditions, whereas the iron-enhanced core is marginally resolved with the radius of $3^{\prime \prime}$. Finally, the $\mathrm{H} \beta$ index profile demonstrates a definite ring located between $R=4^{\prime \prime}$ and $R=8^{\prime \prime}$ with the contrast of $0.6 \AA$, or $40 \%$. What could be an explanation of the ring-like distribution of the $\mathrm{H} \beta$-index? One possibility can be related to Balmer emission contamination. If the ionized gas emission is peaked in the nucleus, it affects the measurement of the nuclear $\mathrm{H} \beta$ absorption-line index (which is in fact an equivalent width) more severely than off-nuclear ones; then in the nucleus the $\mathrm{H} \beta$-index should be more underestimated than beyond the nucleus. We can try to correct the emission effect. Equivalent widths of the azimuthallyaveraged $\mathrm{H}_{\alpha}$ emission line, which is much more intense than the $\mathrm{H}_{\beta}$ emission, can be easily measured because the $\mathrm{H}_{\alpha}$ emission line is narrower than the absorption one. We have done it having obtained $E W\left(\mathrm{H}_{\alpha} \mathrm{em}\right)$ between $0.7 \AA$ and $1.5 \AA$; then we have calculated the equivalent widths of the $\mathrm{H}_{\beta}$ emission line that is exactly the correction of the $\mathrm{H} \beta$-index for the emission, by using recent results of 


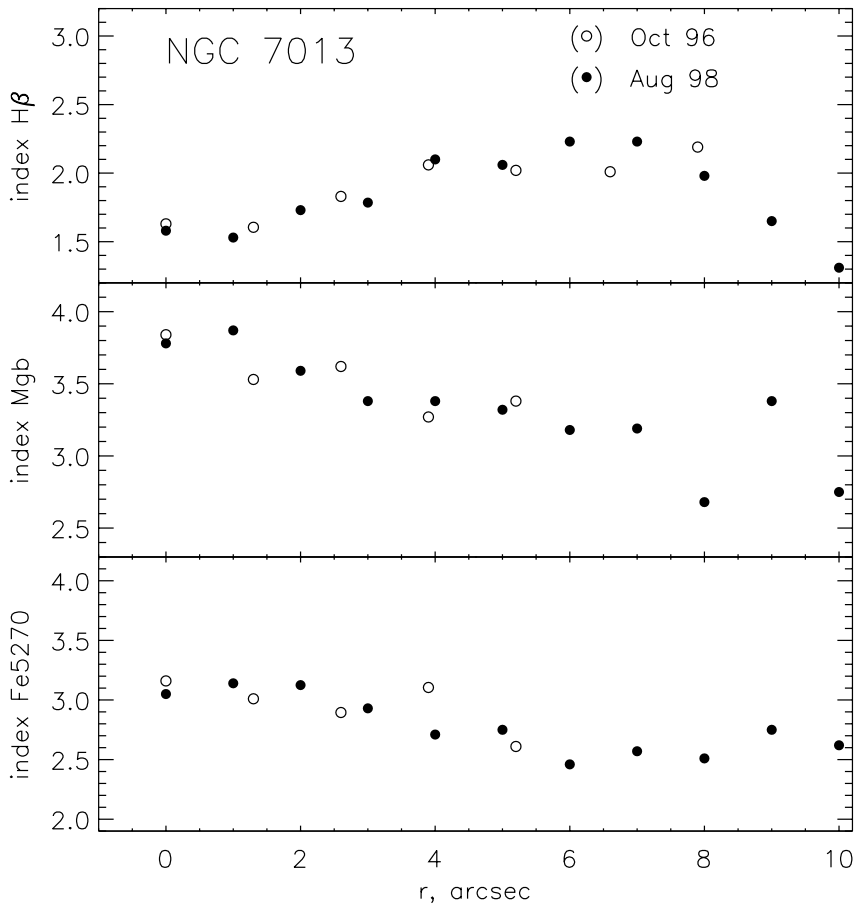

Fig. 4. Radial profiles of the azimuthally-averaged Lick indices in NGC 7013 according to two independent observation runs.

Stasinska \& Sodre (2001) who found an excellent correlation $E W\left(\mathrm{H}_{\beta}\right)=0.25 E W\left(\mathrm{H}_{\alpha}\right)$ for integrated emission in a morphologically heterogeneous sample of nearby galaxies. In principle, the maximum possible correction for the $\mathrm{H}_{\beta}$ emission can be obtained by postulating a pure recombination mechanism of its radiation, in other words, the present star formation effect; in this case the $\mathrm{H}_{\alpha}$-to- $\mathrm{H}_{\beta}$ emission ratio is about 2.5 (e.g. Burgess 1958). For collision excitation this ratio is higher and so the correction for the $\mathrm{H}_{\beta}$ emission is smaller. Though within our range of $E W\left(\mathrm{H}_{\alpha} \mathrm{em}\right)$ in NGC 7013 the difference between the corrections calculated by adopting a recombination mechanism and by taking the prescription of Stasinska \& Sodre (2001) is insignificant, we have plotted the "overcorrected" $\mathrm{H} \beta$ index data, with the corrections of $0.4 E W\left(\mathrm{H}_{\alpha} \mathrm{em}\right)$, in Fig. 5, bottom, together with the uncorrected ones, to show the whole effect on the age determinations. The corrected absorption-line index $\mathrm{H} \beta$ are used to determine mean ages of the stellar populations. If we underestimate $\mathrm{H}_{\alpha}$ emission equivalent widths due to an underlying absorption effect, the ages may be slightly overestimated.

In Fig. 5 we present diagnostic diagrams "index vs. index" for the central part of NGC 7013. Before trying to determine an age, we must check the magnesium-toiron ratio. The population synthesis models by Worthey (Worthey 1992) have shown that in the "Fe5270 vs. Mgb" diagram all the effects including age, initial mass function, and global metallicity move the stellar population along a narrow locus; any deviation from the model locus for $[\mathrm{Mg} / \mathrm{Fe}]=0$ must be treated as a non-solar magnesiumto-iron ratio. For example, most luminous ellipticals have
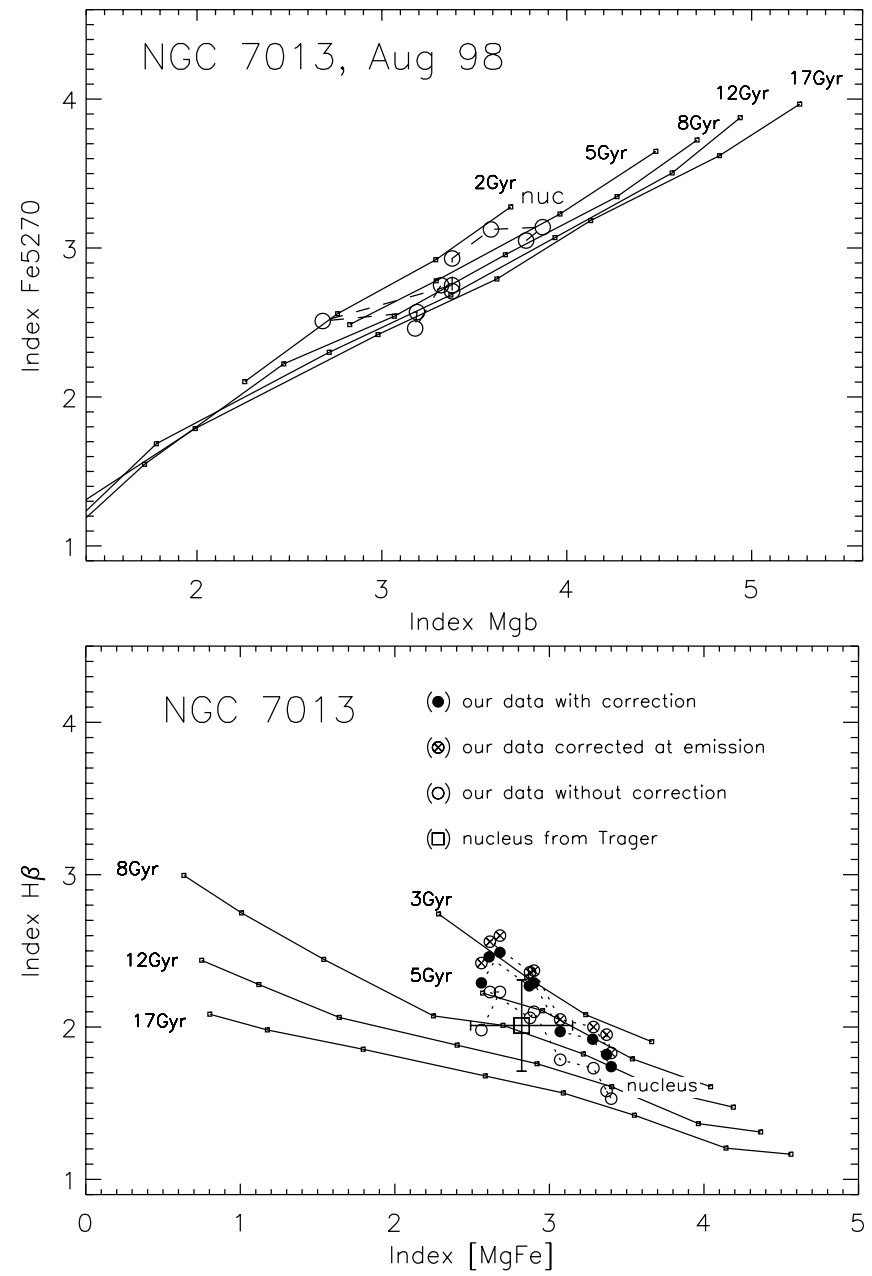

Fig. 5. Index-index diagrams for the azimuthally-averaged, emission-corrected Lick indices taken along the radius with a step of $1^{\prime \prime}$ in the center of NGC 7013 (the large signs connected by dashed lines): (top) Fe5270 vs. Mgb diagram, with the models of Worthey (1994) for $[\mathrm{Mg} / \mathrm{Fe}]=0$, and (bottom) $\mathrm{H} \beta$ vs. $[\mathrm{MgFe}] \equiv(\mathrm{Mgb}<\mathrm{Fe}>)^{1 / 2}$, with the same models and the measurements of Trager et al. (1998) for the $2^{\prime \prime} \times 4^{\prime \prime}$ aperture as a reference. On the lower diagram we show the measurements corrected for the $\mathrm{H} \beta$ emission by black dots and the uncorrected measurements below and the overcorrected ones above (see the text) by open signs. The small signs connected by thin lines represent stellar population models of equal ages; the signs marks the metallicities decreasing from right to left: the metallicities for Worthey's models are $+0.50,+0.25,0.00$, $-0.22,-0.50,-1.00,-1.50,-2.00$.

appeared to be magnesium overabundant (Worthey et al. 1992). As for the central part of NGC 7013, Fig. 5, top, assures us that over the whole central region of NGC 7013 the approximately solar magnesium-to-iron ratio remains. This means that we can use the models of Worthey (1994) that are calculated for the solar $\mathrm{Mg} / \mathrm{Fe}$, to determine the mean ages of the stellar populations at various distances from the center. Figure 5, bottom, shows a diagram of "metal vs. hydrogen" indices and the radial trend of the stellar population properties in the circumnuclear region 

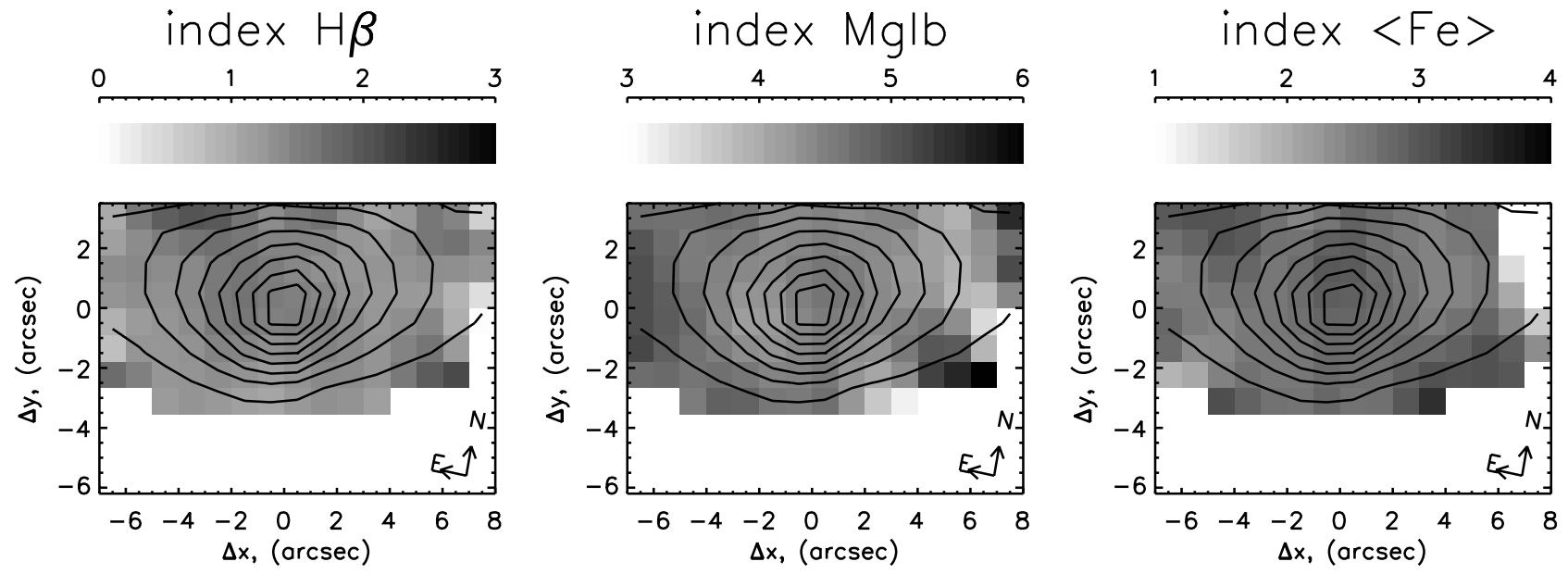

Fig. 6. Lick index maps for the central part of NGC $4429 ;<\mathrm{Fe}>\equiv(\mathrm{Fe} 5270+\mathrm{Fe} 5335) / 2$.

of the galaxy. One can see that in the nucleus the metallicity is slightly supersolar $(\sim+0.1$ dex $)$ with a mean stellar age of 6-8 Gyr; in a few arcseconds from the nucleus the metallicity drops by $0.2-0.3$ dex, but at $R=4^{\prime \prime}$ rises again to the nearly solar value being accompanied by an age decrease down to 3 Gyr. Therefore we must conclude that even after correcting the $\mathrm{H} \beta$-index for the emission we continue to detect a young stellar ring - a zone between $R=4^{\prime \prime}$ and $R=8^{\prime \prime}$ which is distinguished by the lower, with respect to the bulge and to the nucleus, mean age of the stellar population.

\subsection{NGC 4429}

This is another example of the evolutionary distinct circumnuclear ring, though less impressive compared to NGC 7013. Figure 6 presents two-dimensional maps of the absorption-line indices in the central part of NGC 4429. The metal-line indices demonstrate weak enhancement in the nucleus ("chemically distinct nucleus"), perhaps extended along the minor axis. The most prominent feature of both metal-line index maps is a strong enhancement of the magnesium and iron indices at $R>4$ ": the "metalrich" ring is more complete in the magnesium and looks like two separate black spots with a mutual orientation of $\mathrm{PA} \approx 50^{\circ}$ in the iron. The intermediate radii, $R=2^{\prime \prime}-4^{\prime \prime}$, represent a ring of minimum metal-line indices; again, is it a bulge? The $\mathrm{H} \beta$-index map is similar to the $<\mathrm{Fe}>$ map, possessing two "black" spots at $R \approx 6^{\prime \prime}$ close to $\mathrm{PA} \approx 50^{\circ}$; but here one does not see the complete "minimum-index ring", because something like a bar of enhanced $\mathrm{H} \beta$ absorption lies between these spots. All the structures are of rather low contrast, so to quantify their significance we have calculated mean values of the indices over the ring zones at various distances from the center (by using the maps of Fig. 6) and have plotted them in Fig. 7 together with the azimuthally averaged measurements obtained in our traditional manner, i.e. firstly co-adding the spectra over the rings and only after that measuring the indices.
The latter method is more accurate and gives more extended profiles, but the formal errors of the means provided by the former method, compared to the statistical error estimations, enable us to check the axial symmetry of the index distributions. Just as we hoped, for NGC 4429 two methods of measuring the azimuthally-averaged indices gave consistent results, and one can see from Fig. 7 that there is a ring of enhanced indices with the middle at $R \approx 6^{\prime \prime}$ that is quite significant in $\mathrm{H} \beta$, rather significant in Mgb, especially in Mgb measured by the second method, and marginally significant in Fe5270. So, trying to diagnose properties of the stellar populations in the center of NGC 4429, we would consider three substructures: the "nucleus" $-R=0^{\prime \prime}-1$ ", the "bulge" $-R=2^{\prime \prime}-3^{\prime \prime}$, and the "Mg-rich ring" $-R=4^{\prime \prime}-6$ ".

Figure 8 presents the diagnostic diagrams: " $<\mathrm{Fe}>$ vs. Mgb" and "H $\beta$ vs. $<\mathrm{Fe}>$ ". From the former one can conclude that unlike the case of NGC 7013, all three substructures in the center of NGC 4429 are magnesiumoverabundant; above that, the "nucleus" is the most metal-rich, the "bulge" is the least metal-rich (less abundant by some 0.2 dex than the nucleus), and the "ring" lies between them. After evaluating the magnesium-toiron ratio in the center of NGC 4429 by using the $<\mathrm{Fe}>$ shift at a constant magnesium index level with respect to the $[\mathrm{Mg} / \mathrm{Fe}]=0$ (Tantalo et al.'s 1998 model), we choose their model with $[\mathrm{Mg} / \mathrm{Fe}]=+0.3$ to estimate the ages of the stellar populations. The diagram "H $\beta$ vs. $<\mathrm{Fe}>$ " shows that the chemically distinct nucleus of the lenticular galaxy NGC 4429 is rather young: its mean stellar age is 7-8 Gyr. Our measurements of the equivalent width of the $\mathrm{H}_{\alpha}$ emission line in the center of NGC 4429 have shown that it is everywhere less than $0.5 \AA$, so the $\mathrm{H} \beta$-index correction for the emission should be everywhere not larger than $0.1 \AA$ and so can be omitted. Therefore we must treat an increase of the mean age of the stellar population along the radius up to $R=4^{\prime \prime}-5^{\prime \prime}$ as a real effect. But at the outer edge of the "Mg-rich ring", at $R=6^{\prime \prime}-7$ " (see also Fig. 7), the mean stellar population age drops again and 


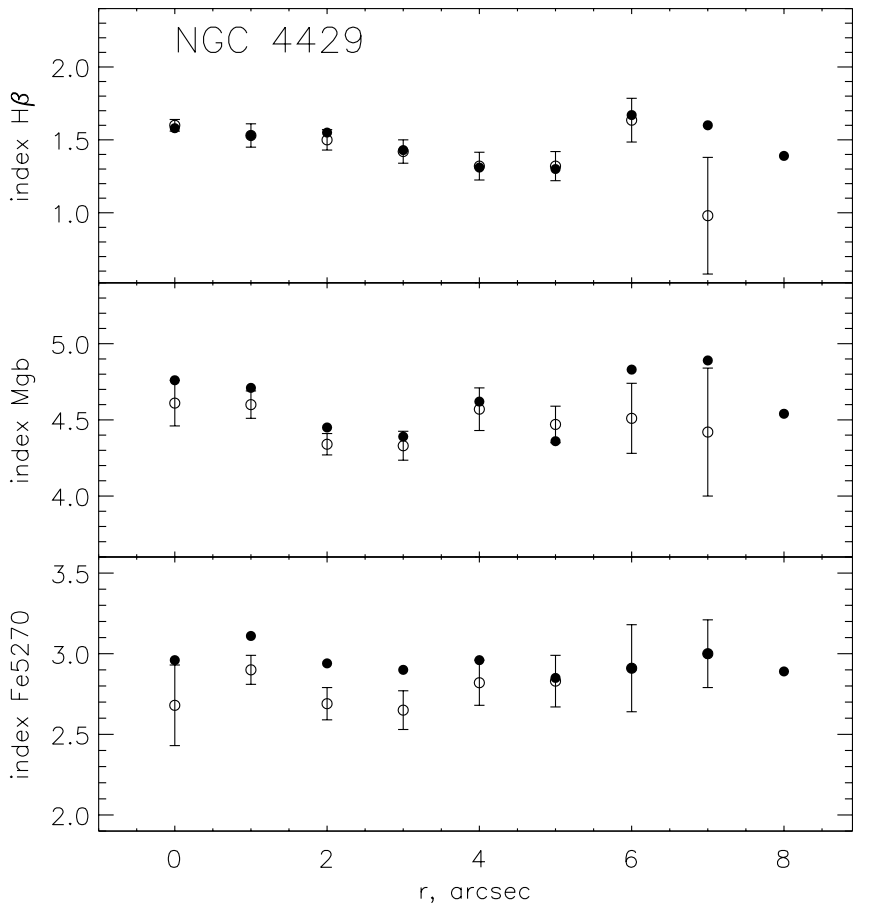

Fig. 7. Radial profiles of the azimuthally-averaged Lick indices in NGC 4429 obtained by two different methods as described in the text.

is probably close to the age of the nuclear stellar population. In other words, there is another young stellar ring, though not so young as that in NGC 7013.

\section{Kinematics of gas and stars in the centers of NGC 4429 and NGC 7013}

Since the integral-field spectroscopy provides us with two-dimensional line-of-sight velocity fields, we are now able to analyse both the character of rotation and axisymmetry of the central structure components. If we have an axisymmetric mass distribution and rotation on circular orbits, the direction of the maximum central line-of-sight velocity gradient (we shall call it "kinematical major axis") should coincide with the line of nodes as well as the photometric major axis; whereas in the case of triaxial potential the isovelocities align with the principal axis of the ellipsoid, and generally the kinematical and photometric major axes diverge, showing turns with respect to the line of nodes in opposite senses (Monnet et al. 1992; Moiseev \& Mustsevoy 2000). In the simple case of cylindric (disk-like) rotation we have a convenient analytical expression for the azimuthal dependence of central line-of-sight velocity gradients within the area of solid-body rotation:

$\mathrm{d} v_{\mathrm{r}} / \mathrm{d} r=\omega \sin i \cos \left(\mathrm{PA}-\mathrm{PA}_{0}\right)$

where $\omega$ is the deprojected central angular rotation velocity, $i$ is the inclination of the rotation plane, and $\mathrm{PA}_{0}$ is the orientation of the line of nodes, coinciding in the
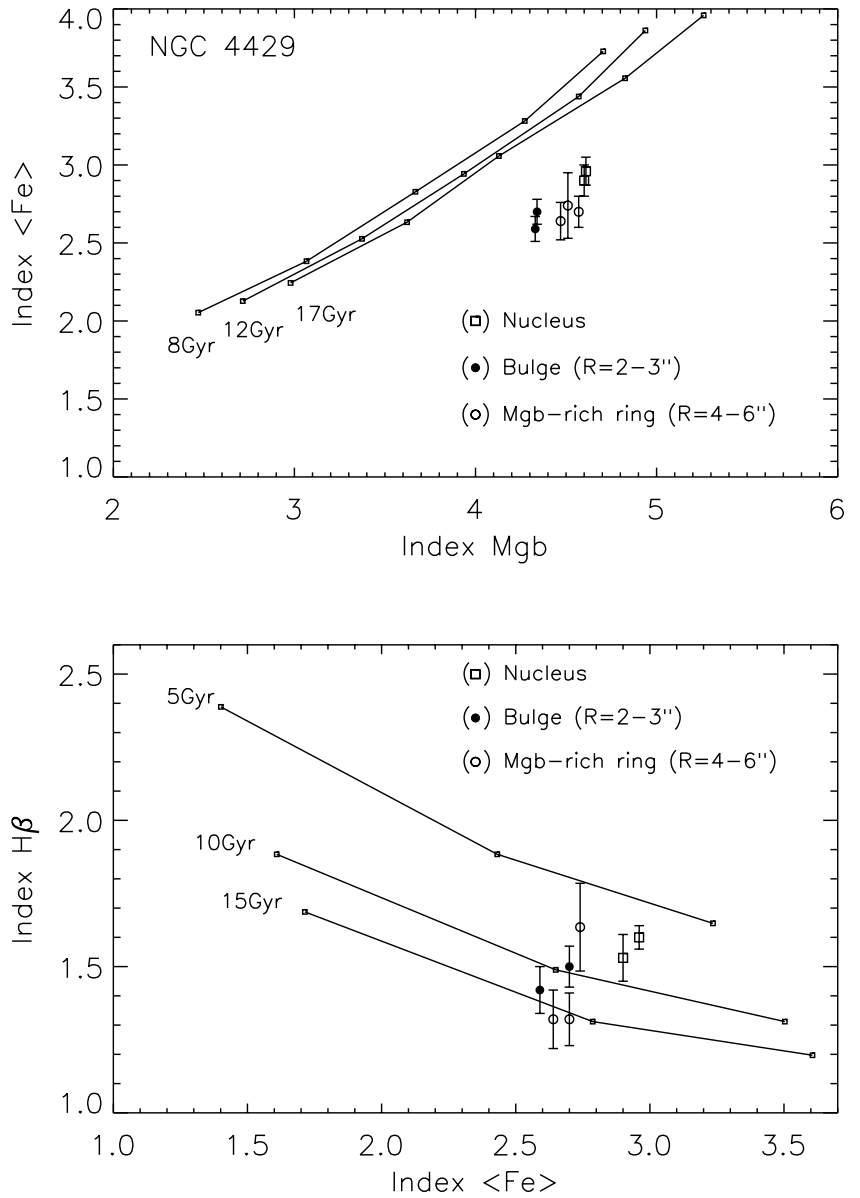

Fig. 8. Index-index diagrams for the azimuthally-averaged Lick indices taken along the radius with a step of $1^{\prime \prime}$ in the center of NGC 4429 (large signs with error bars); (top) $<\mathrm{Fe}>$ vs. Mgb diagram, with the models of Worthey (1994) for $[\mathrm{Mg} / \mathrm{Fe}]=0$, and (bottom) $\mathrm{H} \beta$ vs. $<\mathrm{Fe}>$, and the models of Tantalo et al. (1998) for $[\mathrm{Mg} / \mathrm{Fe}]=+0.3$. Small signs connected by thin lines represent stellar population models of equal ages; the metallicities for Worthey's models are +0.50 , $+0.25,0.00,-0.22,-0.50,-1.00,-1.50,-2.00$, if one takes the signs from right to left, and for the models of Tantalo et al. they are $+0.4,0.0$, and -0.7 .

case of an axisymmetric ellipsoid (or a thin disk) with the photometric major axis. So by fitting azimuthal variations of the central line-of-sight velocity gradients with a cosine curve, we can determine the orientation of the kinematical major axis by its phase and the central angular rotation velocity by its amplitude. This is our main tool of kinematical analysis.

Figure 9 presents velocity fields for the ionized gas and stars in the center of NGC 4429 as well as a stellar velocity dispersion map. The LOS velocity fields look quite regular, similar to each other, and reflect probably pure rotation with a local maximum of the rotation velocity about $5^{\prime \prime}$ from the center. The stellar velocity dispersion map is not so common: instead of the (unresolved) maximum in the nucleus or featureless flat distribution one can see an extended, rather low-contrast off-nuclear maximum to the north-west of the center. 

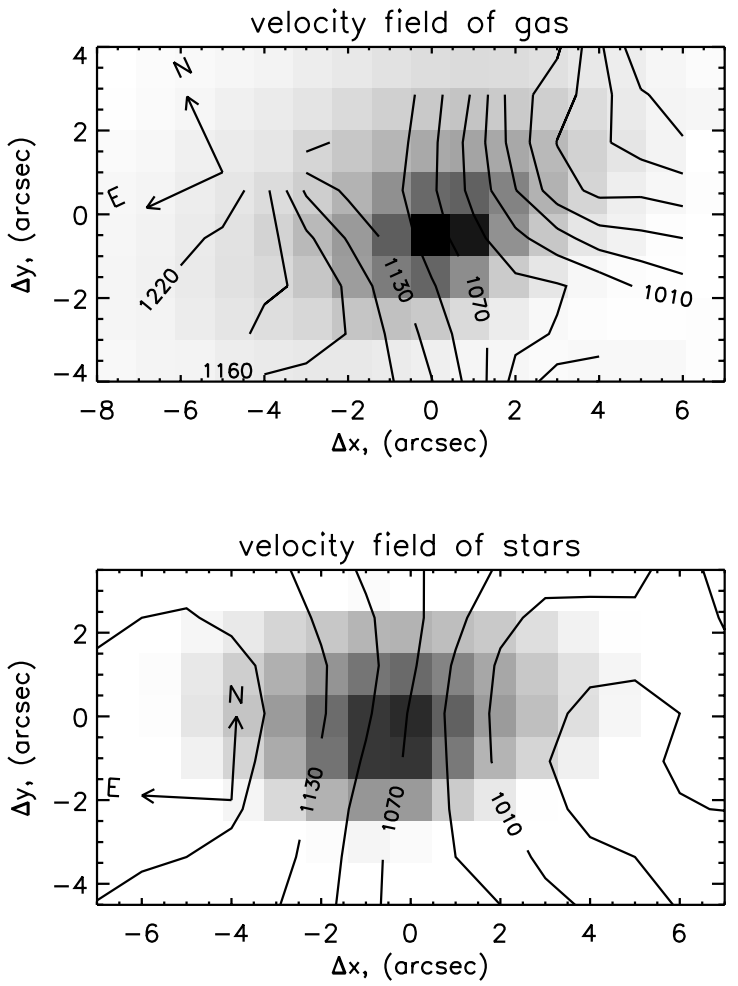

velocity dispersion of stars, $\mathrm{km} / \mathrm{s}$

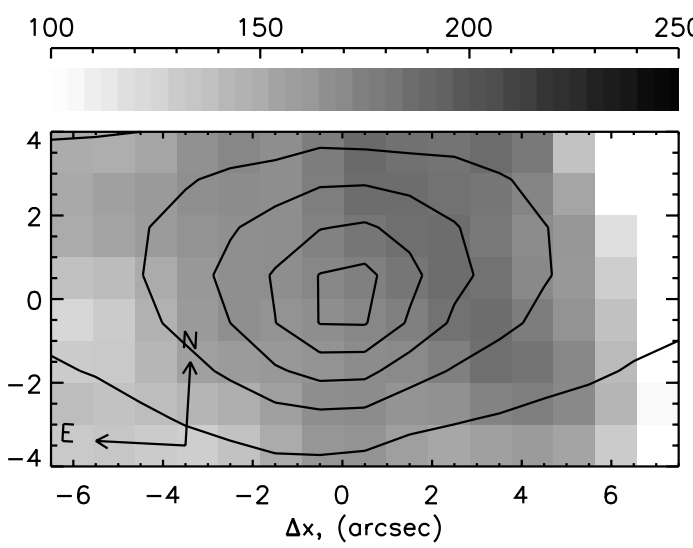

Fig. 9. Line-of-sight velocity fields for the ionized gas and stars (isolines overlaid onto the gray-scaled continuum map) and the stellar velocity dispersion map (gray-scaled, with the continuum isophotes overlaid) in the center of NGC 4429.

In Fig. 10 we compare the orientations of the kinematical and photometric (from the various archive sources) major axes. At $R>2^{\prime \prime}$ the estimation of the kinematical major axes both for the stars and ionized gas is quite consistent with the global line of nodes of the galaxy and with the isophote orientation at these radii. (We note that our value of $\mathrm{PA}_{0}$ for the line of nodes obtained from the outermost isophote orientation at $R>160^{\prime \prime}$, namely, $\mathrm{PA}_{0}=89^{\circ}$, differs by $10^{\circ}$ from the RC3 value, but agrees perfectly with the recent result of Koopmann et al. 2001.) So at $R>2^{\prime \prime}$ all the constituents rotate axisymmetrically, with the rotation plane being parallel to the main symmetry plane of the galaxy. Inside $R=11^{\prime \prime} 7$ - and our seeing

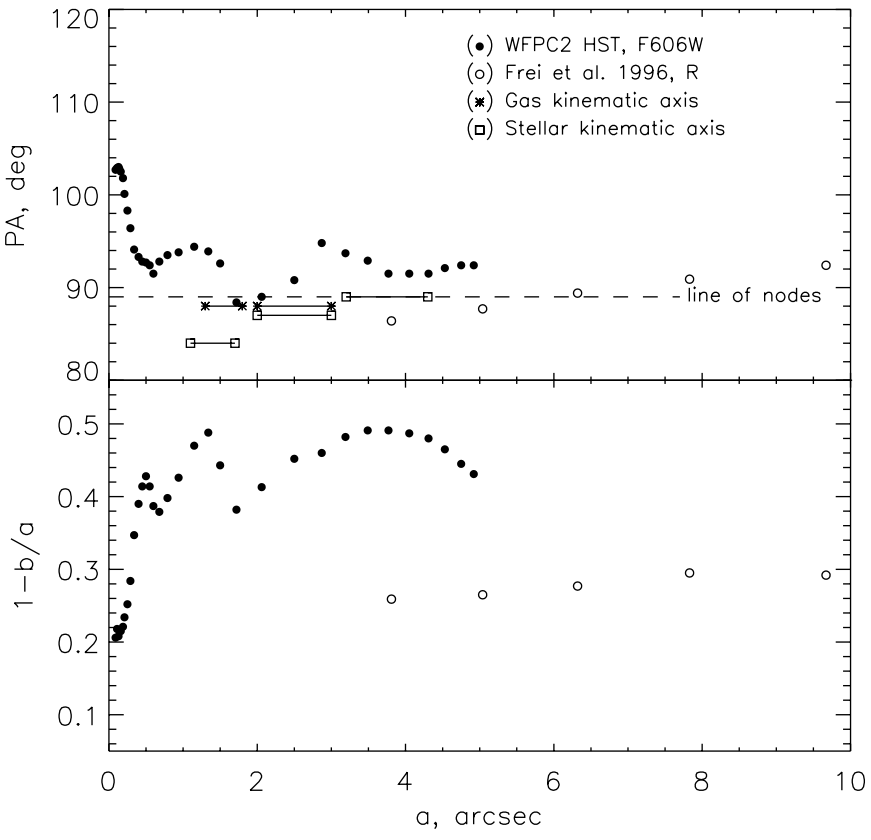

Fig. 10. Isophote characteristics together with the orientations of the kinematical major axes for the stars and ionized gas in the center of NGC 4429.

quality of $1^{\prime \prime} .5$ gives us a hope that this result is relevant we observe a turn of the kinematical major axis by $5^{\circ}$ in the counter-direction to the turn of the photometric major axis by the same $5^{\circ}$. Such mirror deviations suggest an elliptical rotation in the non-axisymmetric potential; the turn of the photometric major axis inside $R=0.5$ implied by the HST data supports a very compact triaxial structure in the center of NGC 4429 (unfortunately, we have no kinematics with such high resolution).

Finally, we have constructed two-dimensional maps of the gas emission surface brightness distributions (Fig. 11). Both $\mathrm{H}_{\alpha}$ and $[\mathrm{N} \mathrm{II}] \lambda 6583$ emissions possess disk-like distributions, elongated close to the line of nodes. However, a prominent difference also exists: the [N II] emission distribution is more centrally concentrated, whereas the $\mathrm{H}_{\alpha}$ emission is not only more extended, but also demonstrates two off-nuclear peaks symmetrically located on the major axis in $4^{\prime \prime}$ from the center. It may trace sites of current star formation at the inner edge of the "Mg-rich ring". A small difference also exists in the orientations of "[N II]-disk" and " $\mathrm{H}_{\alpha}$-disk": while the latter is elongated in $\mathrm{PA}=89^{\circ}$, exactly along the line of nodes, the former has the isophote major axis in $\mathrm{PA} \approx 98^{\circ}$, or turned following the probable nuclear bar orientation. This may be accounted for if a significant fraction of the [N II] emission originates from shock waves near the edges of the minibar, and $\mathrm{H}_{\alpha}$ is mainly related to the star formation in the circumnuclear disk (ring).

Figure 12 presents two-dimensional velocity fields for the ionized gas and stars in the center of NGC 7013 as well as the stellar velocity dispersion map. All three maps look like twins of the maps in Fig. 9 for NGC 4429: both stars 

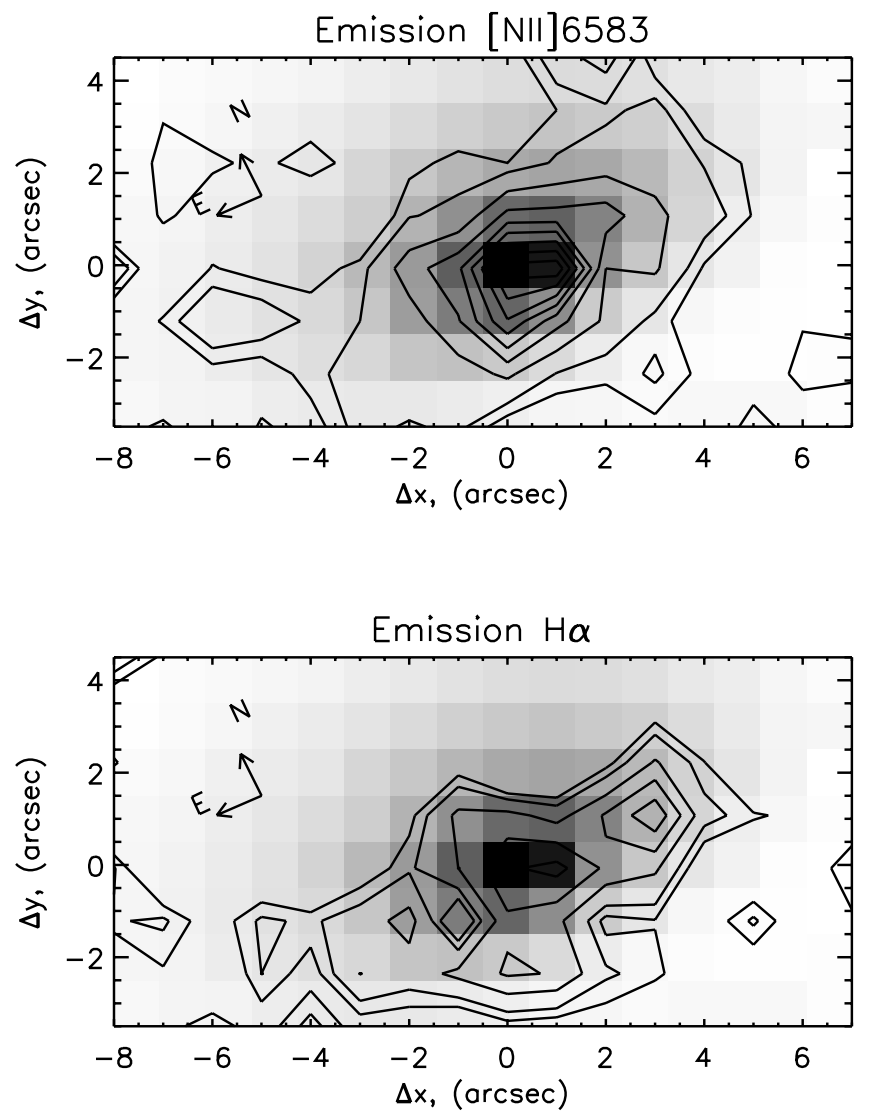

Fig. 11. Surface emission-line brightness distributions in the center of NGC 4429 (isolines overlaid onto the gray-scaled red continuum).

and gas rotate regularly, with local maxima of rotation velocity at $R \approx 5^{\prime \prime}$, and the stellar velocity dispersion demonstrates an arc-like maximum at the same $R \approx 5^{\prime \prime}$ north of the nucleus. Now we can identify the half-ring of the maximum velocity dispersion with the ring of the enhanced $\mathrm{H} \beta$ absorption-line index (Fig. 3).

However, the analysis of the kinematical major axis orientations in the center of NGC 7013 (Fig. 13) gives quite different results if compared to Fig. 10. While at $R>3^{\prime \prime}$ the ionized gas and stars in NGC 7013 rotate axisymmetrically in the main symmetry plane of the galaxy, inside the radius of $3^{\prime \prime}$ the kinematical and photometric major axes turn consistently to larger values of PA. This consistency means that we deal with an inclined circumnuclear disk. The HST isophote ellipticity demonstrates a prominent local maximum at $R=3^{\prime \prime} .5$ - it is probably an outer edge of the circumnuclear disk. Inside $R \approx 2^{\prime \prime}$ the photometric data does not support the further turn of the kinematical major axis. Perhaps, here the photometric and spectral observations probe different stellar components: measuring stellar velocities by cross-correlation with the star template, we always measure the coldest dynamical subsystem on the line of sight, even if it is a faint disk fully embedded in the bright spheroid; in the meantime we know that near the nucleus of NGC 7013 the dominant photometric component is a de Vaucouleurs' bulge (HST
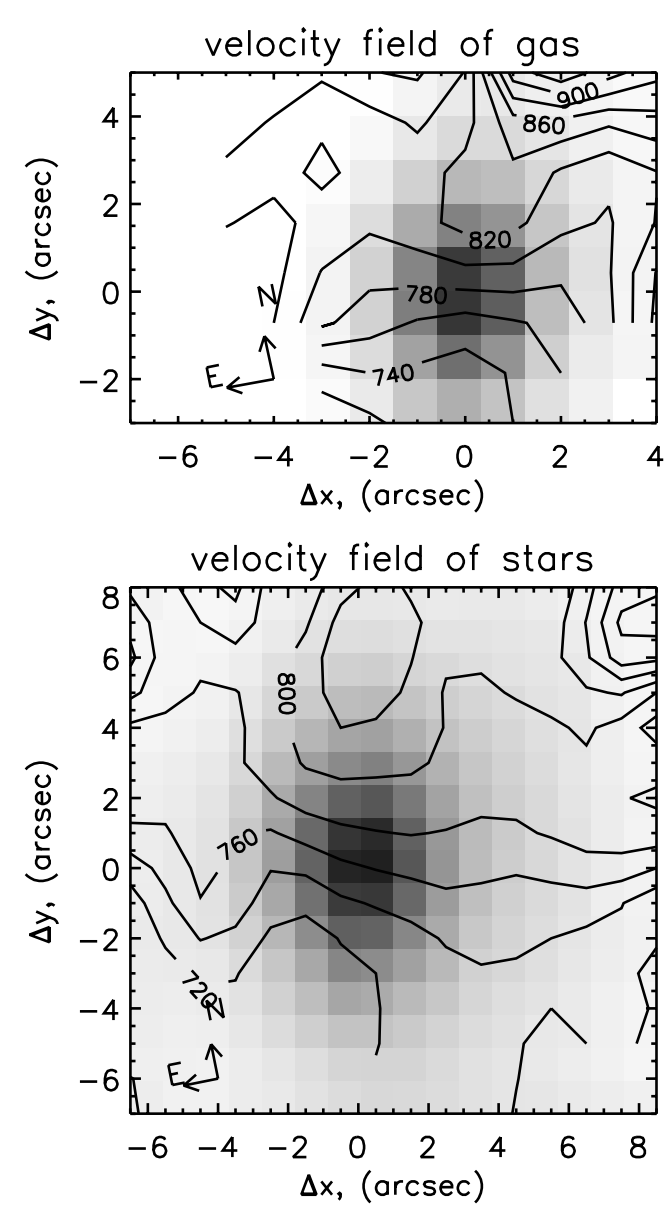

velocity dispersion of stars, $\mathrm{km} / \mathrm{s}$

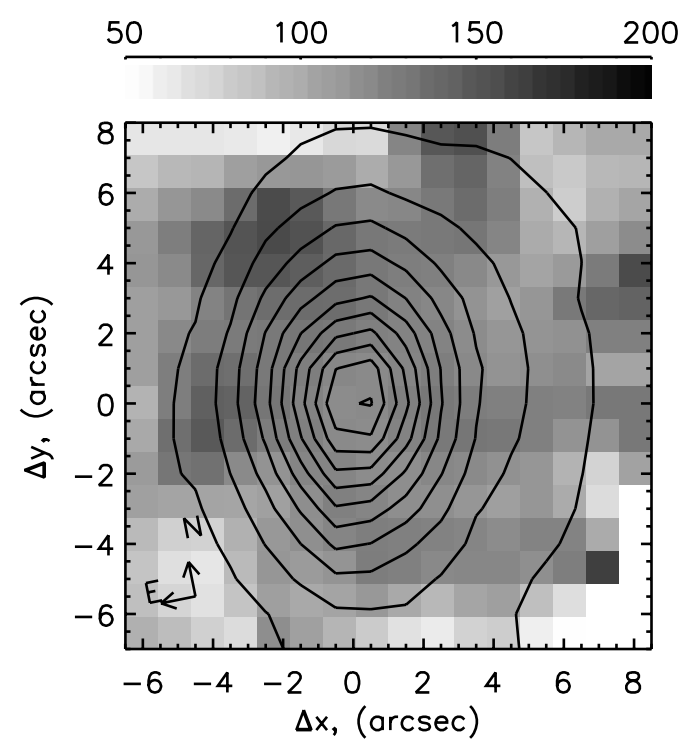

Fig. 12. Line-of-sight velocity fields for the ionized gas and stars (isolines overlaid onto the gray-scaled continuum map) and the stellar velocity dispersion map (gray-scaled, with the continuum isophotes overlaid) in the center of NGC 7013.

data, Carollo et al. 1997). Interestingly, the major axis of the circumnuclear disk, $\mathrm{PA} \geq 172^{\circ}$, has the same orientation as the inner stellar ring at $R \approx 20^{\prime \prime}\left(\mathrm{PA}_{* \text { ring }} \approx 170^{\circ}\right.$, 


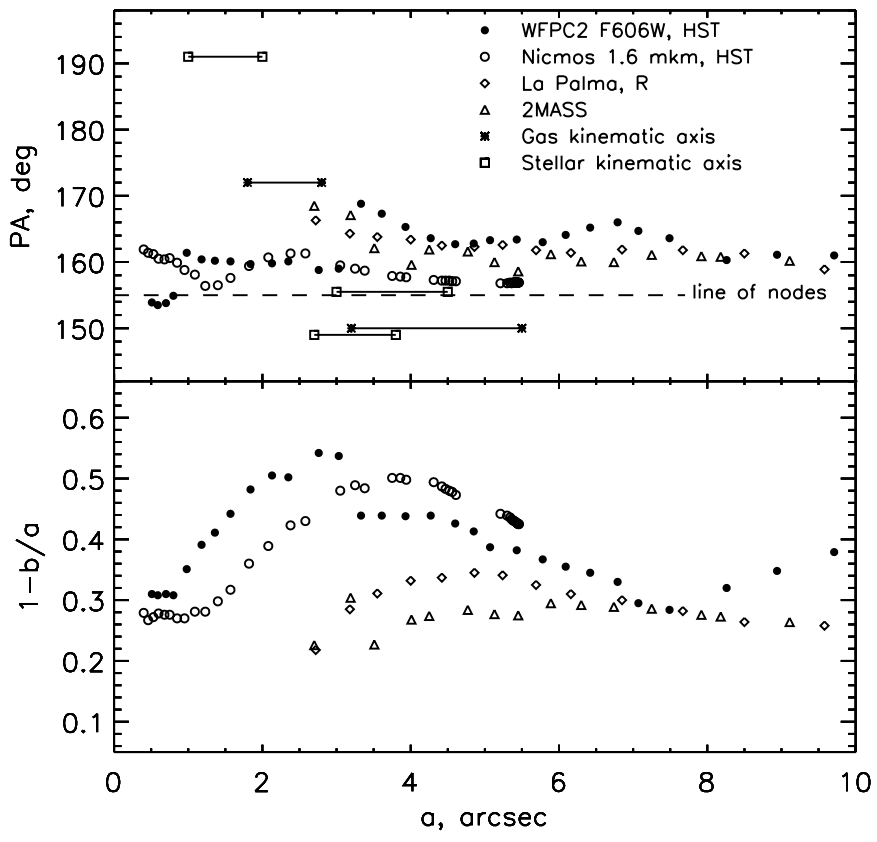

Fig. 13. Isophote characteristics together with the orientations of the kinematical major axes for the stars and ionized gas in the center of NGC 7013

Prieto et al. 2001), and the inner H I ring at $R=40^{\prime \prime}-$ $60^{\prime \prime}\left(\mathrm{PA}_{\mathrm{HI} \text { ring }}=175^{\circ}\right.$, Knapp et al. 1984). Naturally, we suspect here the common origin.

Figure 14 shows the surface distributions of the emission-line intensities in the center of NGC 7013. The [N II] $\lambda 6583$ emission is strongly concentrated in the center; unlike in NGC 4429, it does not reveal any hints about linear shock waves usually related to the edges of bars. The $\mathrm{H}_{\alpha}$ emission is more extended, but the appearance of its brightness distribution is rather chaotic, and we cannot find any relation between the shape of $\mathrm{H}_{\alpha}$ emission isophotes and other factors.

\section{Discussion}

We have found a new type of chemically distinct galactic nucleus: a ringed core. This consists of a central compact structure - the "unresolved nucleus" - and a separate ring with a radius of a few hundred parsecs. Some time ago, when we investigated chemically distinct nuclei in NGC 1023 and NGC 7331, we found that they were in fact circumnuclear disks consisting of a magnesium overabundant nucleus with a stellar population of intermediate age and outskirts that were younger by $2-3$ Gyr and with solar Mg/Fe (Sil'chenko 1999a, 1999b). All these features are consistent with a scenario according to which a secondary star formation burst started simultaneously over an extended central area, exhausted itself in less than $1 \mathrm{Gyr}$ in the nucleus, and continued in the disk for 3-4 Gyr. Analysing this scenario, we suggested that there is a good chance of catching a forming decoupled stellar core at a moment when the nuclear star formation has stopped but
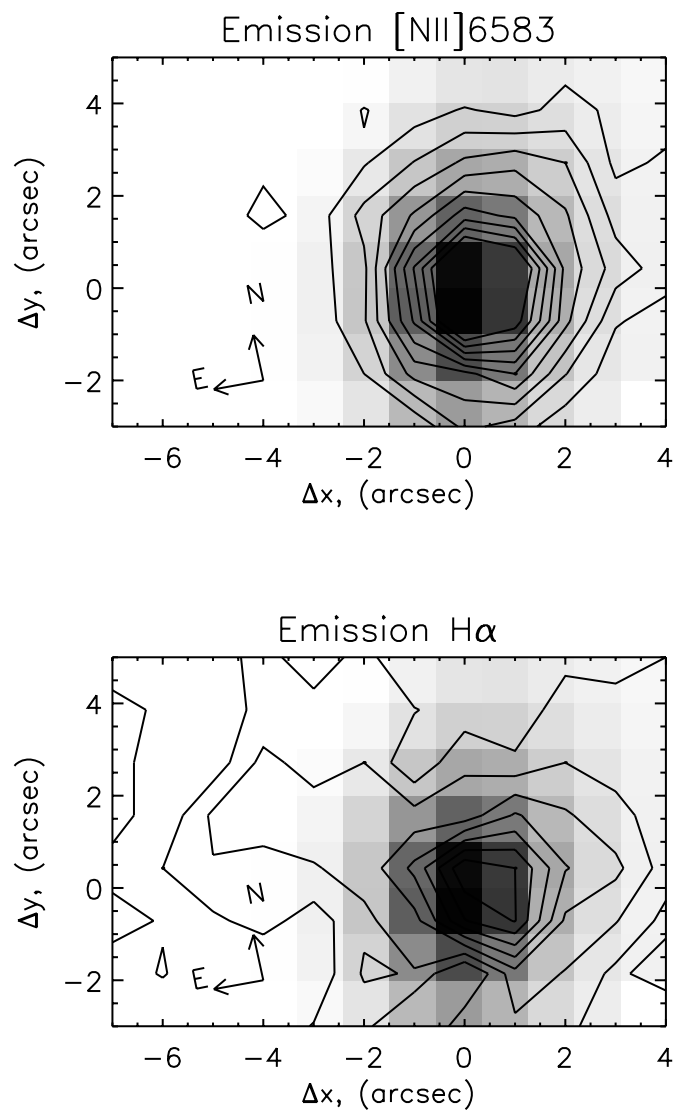

Fig. 14. Surface emission-line brightness distributions in the center of NGC 7013 (isolines overlaid onto the gray-scaled red continuum).

the off-nuclear one still continues. Such an object should look like a circumnuclear H II ring without associated resonance. But NGC 4429 and NGC 7013, although demonstrating a ring shape of star formation burst remnants, cannot be directly built into this scheme. Firstly, unlike NGC 1023 and NGC 7331 where there is a Mg/Fe ratio trend between the nuclei and their outskirts, in NGC 4429 and NGC 7013 the magnesium-to-iron ratios are roughly constant along the radius and are the same in the nuclei and in the rings; it implies comparable durations of the nuclear and off-nuclear star formation bursts. Secondly, and crucially, in NGC 4429 and NGC 7013 there is a gap between the nuclei and the rings, where the stellar population is less chemically evolved than in the nuclei and in the rings. Perhaps we have noticed these gaps because the angular diameters of the rings in NGC 4429 and NGC 7013 are twice the size of that in NGC 7331 and larger by a factor of 4 than in NGC 1023 - with our spatial resolution we should not be able to notice such gaps in the latter two galaxies even if they exist. In any case, the secondary star formation bursts in NGC 4429 and NGC 7013 indeed have a ring geometry, and it remains for us to explain why. Again, we return to the idea of resonances of triaxial structures that may easily produce star-forming rings. 
We mentioned that NGC 4429 and NGC 7013 possess large-scale stellar rings - in addition to the ring-like chemically decoupled cores. Buta (1991), in a final paper on weakly (or non-) barred ringed galaxies, included these two galaxies in the list of prospective objects of this type; in the same work he proposed transient large-scale bars as a possible source of the long-lived stellar rings in earlytype disk galaxies. Indeed, we note some signatures of the past (dissolved) bars in NGC 4429 and NGC 7013: both galaxies have large-scale lenses - structural components intermediate between the bulges and the global disks. As for NGC 4429, there has been only a hint in the literature of its lens (Watanabe et al. 1982), and we have assured ourselves of its presence by subtracting a model image of the global disk from the $R$-band image of the galaxy provided by Frei et al. (1996) (following the approach of Baggett et al. 1998, we have fitted the azimuthally averaged brightness profile in the radius range of $R>100^{\prime \prime}$ by an exponential and have determined the disk parameters: $\mu_{0}=19.8, h=52^{\prime \prime}$. 3 ). The residual brightness image possesses a diffuse constituent extending up to $R \approx 120^{\prime \prime}$ and elongated in $\mathrm{PA} \approx 100^{\circ}$ with an isophote ellipticity of 0.7 . The visible ellipticity of the global disk is 0.5 , the line of nodes is $\mathrm{PA}_{0}=89^{\circ}$, and in the radius range of $30^{\prime \prime}-80^{\prime \prime}$ a pair of thick red spirals can be seen. We conclude that this intermediate component is an oval disk, or a lens. A similar lens with the skewed major axis and high ellipticity of the isophotes has been recently found in NGC 7013 by Prieto et al. (2001). Though the lenses are rather frequent phenomenon, their exact nature has not yet been investigated. But since in our particular case the lenses are obviously oval, we would conclude, following the hypothesis of Kormendy (1979), that they are probably products of bar decay. Whenever there were transient short-lived, large-scale bars in NGC 4429 and NGC 7013, they provoked intense gas inflow into the center of the galaxies that should stop after bar dissolution. It should be these events that produced chemically distinct cores in NGC 4429 and NGC 7013.

NGC 4429 and NGC 7013 look rather similar in their global morphology; but in this investigation we have shown quite different circumnuclear kinematical structures in these galaxies (Sect. 4). Why does NGC 7013 possess a circumnuclear disk, and why does NGC 4429 possess a nuclear bar, if the origin of the circumnuclear structures in these galaxies was the same? We may speculate about the amount of gas in the galaxies. Neither neutral nor molecular gas is found in NGC 4429. As for NGC 7013, it has two large-scale $H$ I rings, each of $(2 \div 3) \times 10^{8} h_{100}^{-2} M_{\odot}$, the inner ring being inclined with the orientation of the major axis $\mathrm{PA} \approx 175^{\circ}$ (Knapp et al. 1984), this is similar to that of the circumnuclear kinematical major axes of stars and gas, and a compact molecular-gas circumnuclear disk of $7 \times 10^{7} M_{\odot}$ (Taniguchi et al. 1994), so it is usually characterized as "a gas-rich S0". By simulating gas inflow due to bar instability in a two-component selfgravitating disk, Shlosman \& Noguchi (1993) have noted that the mass fraction of the gas is crucial: if it is less that
$10 \%$, the bar persists and grows, if it is larger, the bar is damped, and gas clouds fall onto the center due only to dynamical friction that results in an axisymmetric circumnuclear disk. Perhaps this effect is important in our case, and NGC 7013 has no nuclear bar because of its gas richness. NGC 4429 is a member of the Virgo cluster and it might lose a significant fraction of its gas due to ram stripping at the early stage of evolution, its gas paucity has affected the formation of a chemically distinct core, producing a minibar in the circumnuclear stellar disk.

Acknowledgements. We thank the astronomers of the Special Astrophysical Observatory Drs. A. N. Burenkov, S. N. Dodonov, and V. V. Vlasyuk, and the post-graduate student A. V. Moiseev for supporting the observations at the $6 \mathrm{~m}$ telescope; the latter also for help with the manuscript preparation. The $6 \mathrm{~m}$ telescope is operated with the financial support of Science Ministry of Russia (registration number 01-43). During the data analysis we used the LyonMeudon Extragalactic Database (LEDA) supplied by the LEDA team at the CRAL-Observatoire de Lyon (France) and the NASA/IPAC Extragalactic Database (NED) which is operated by the Jet Propulsion Laboratory, California Institute of Technology, under contract with the National Aeronautics and Space Administration. This research has made use of the ING Archive. The JKT is operated on the island of La Palma by the Royal Greenwich Observatory in the Spanish Observatorio del Roque de los Muchachos of the Instituto de Astrofisica de Canarias. The research is also partly based on observations made with the NASA/ESA Hubble Space Telescope, obtained from the data archive at the Space Telescope Science Institute, which is operated by the Association of Universities for Research in Astronomy, Inc., under NASA contract NAS 5-26555. The work was supported by the grant 01-02-16767 of the Russian Foundation for Basic Researches and by the Russian State ScientificTechnical Program "Astronomy. Basic Space Researches" (the "Astronomy" section), the grant 1.2.4.1.

\section{References}

Afanasiev, V. L., Dodonov, S. N., Drabek, S. V., \& Vlasyuk, V. V. 1996, MPFS Manual (Nizhnij Arkhyz: SAO Publ.)

Athanassoula, E. 1996, in Barred Galaxies, ed. R. Buta, D. A. Crocker, \& B. G. Elmegreen, ASP Ser., 91, 309

Bacon, R., Adam, G., Baranne, A., et al. 1995, A\&AS, 113, 347

Baggett, W. E., Baggett, S. M., \& Anderson, K. S. J. 1998, AJ, 116, 1626

Burgess, A. 1958, MNRAS, 118, 477

Buta, R. 1991, ApJ, 370, 130

Buta, R. 1993, PASP, 105, 654

Buta, R., \& Crocker, D. A. 1993, AJ, 105, 1344

Buta, R., van Driel, W., Braine, J., et al. 1995, ApJ, 450, 593

Cardiel, N., Gorgas, J., Cenarro, J., \& Gonzalez, J. J. 1998, A\&AS, 127, 597

Carollo, C. M., Stiavelli, M., de Zeeuw, P. T., \& Mack, J. 1997, AJ, 114, 2366

Frei, Z., Guhathakurta, P., Gunn, J. E., \& Tyson, J. A. 1996, AJ, 111, 174

Friedli, D., \& Benz, W. 1993, A\&A, 268, 65

Heller, C. H., \& Shlosman, I. 1994, ApJ, 424, 84 
Knapp, G. R., van Driel, W., Schwarz, U. J., van Woerden, H., \& Gallagher III, J. S. 1984, A\&A, 133, 127

Koopmann, R. A., Kenney, J. D. P., \& Young, J. 2001, ApJS, 135,125

Kormendy, J. 1979, ApJ, 227, 714

Moiseev, A. V., \& Mustsevoy, V. V. 2000, Pis'ma v AZh, 26, 657

Monnet, G., Bacon, R., \& Emsellem, E. 1992, A\&A, 253, 366

Noguchi, M. 1988, A\&A, 203, 259

Prieto, M., Aguerri, J. A. L., Varela, A. M., \& Munoz-Tunon, C. 2001, A\&A, 367, 405

Shlosman, I., \& Noguchi, M. 1993, ApJ, 414, 474

Sil'chenko, O. K. 1994, AZh, 71, 706

Sil'chenko, O. K. 1999a, AJ, 117, 2725

Sil'chenko, O. K. 1999b, AJ, 118, 186

Sil'chenko, O. K., Afanasiev, V. L., \& Vlasyuk, V. V. 1992, AZh, 69, 1121
Stasinska, G., \& Sodre Jr., I. 2001, A\&A, 374, 919

Taniguchi, Y., Murayama, T., Nakai, N., Suzuki, M., \& Kameya, O. 1994, AJ, 108, 468

Tantalo, R., Chiosi, C., \& Bressan, A. 1998, A\&A, 333, 419

Thakar, A. R., \& Ryden, B. S. 1998, ApJ, 506, 93

Trager, S. C., Worthey, G., Faber, S. M., Burstein, D., \& Gonzalez, J. J. 1998, ApJS, 116, 1

Tremaine, S., \& Yu, Q. 2000, MNRAS, 319, 1

Vlasyuk, V. V. 1993, Astrofiz. issled. (Izv. SAO RAS), 36, 107

Watanabe, M., Kodaira, K., \& Okamura, S. 1982, ApJS, 50, 1

Worthey, G. 1992, Ph.D. Thesis, Univ. of California, Santa Cruz

Worthey, G. 1994, ApJS, 95, 107

Worthey, G., Faber, S. M., \& Gonzalez, J. J. 1992, ApJ, 398, 69

Worthey, G., Faber, S. M., Gonzalez, J. J., \& Burstein, D. 1994, ApJS, 94, 687 